\title{
A SURVEY FOR ULTRAVIOLET-EXCESS GALAXIES WITH THE KISO SCHMIDT TELESCOPE
}

\author{
B. TAKASE ${ }^{1}$ and N. MIYAUCHI-ISOBE ${ }^{2}$ \\ ${ }^{1}$ Kokugakuin University, Shibuyaku, Tokyo, Japan \\ 2 National Astronomical Observatory of Japan, Mitaka, Tokyo, Japan
}

\begin{abstract}
We have detected 8,968 ultraviolet-excess galaxies in a sky area of some 5,100 square degrees by means of the UGR three-image photographs with the $105 \mathrm{~cm}$ Kiso Schmidt, giving a number density of 1.8 per square degree. A net count of the objects is 8,162 , deleting those which appear repeatedly in the neighbouring survey area. For each of these galaxies which we call KUGs, data including morphological type and UV-excess degree are listed in a catalogue series of 18 volumes. Some statistics of these KUGs are also given in the last volume.
\end{abstract}

\section{Survey}

Our survey for ultraviolet-excess galaxies is based on UGR (or UR) three (or two)-colour photographs taken with the $105 \mathrm{~cm}$ Kiso Schmidt telescope. Exposure times are set such that these colour images of A0 stars are equally bright on the plate. In all, 8,968 UV-excess galaxies have been detected in a sky area of some 5,100 square degrees. So the number density of these galaxies which we call KUGs is 1.8 per square degree. After deleting those which appear repeatedly in the neighbouring survey areas, a net count of the objects results in 8,162. The limiting magnitude of a survey plate varies from 17 to 18.5 depending on the plate quality and observational conditions.

\section{The KUG Catalogue}

The catalogue is composed of 18 volumes, 17 of which list coordinates, morphological type, size, magnitude and UV-excess degree, together with other catalogue names, if any, of the KUGs detected in each ten sky areas (e.g. Takase \& Miyauchi-Isobe 1993a). The finding chart for every KUG is also given. The last catalogue, Vol. 18 (Takase \& Miyauchi-Isobe 1993b), is a merged catalogue of all KUGs, arranged in order of right ascension, with some general descriptions and statistics of the data.

In the following is the meaning of each designation of the morphological types (Takase et al. 1983):

Ic Irregular with clumpy HII regions

Ig Irregular with a giant HII region 
Pi Pair of interacting components

Pd Pair of detached components

Sk Spiral with knotty arms

Sp Spiral with peculiar bar and/or nucleus

C Compact.

When the type is not certainly assigned a colon (:) is attached, and when unclassifiable a question mark (?) is given.

As to the UV-excess degree, $\mathrm{H}, \mathrm{M}$ and $\mathrm{L}$ mean high, medium and low, roughly corresponding to $\mathrm{U}-\mathrm{B} \leq-0.5, \sim-0.3$, and $\sim 0$, respectively (Takase et al. 1983: Noguchi et al. 1980).

\section{Statistics}

The frequency distribution of 8,162 KUGs in respect of morphological type and UV-excess is shown in Fig. 1.

We see that Sp (including Sp:, and this convention is repeated in all cases following) type is most numerous, $44 \%$ of all the sample, which is followed by Sk (19\%) and C (15\%) types, while other types (Ic, Ig, Pi and Pd) are all less than 3\%. The distribution in the UV-excess degree is quite different from type to type. The fraction of $\mathrm{H}$ is highest for Ig (54\%), higher for Ic (33\%) and Pi (22\%), while that of $\mathrm{M}$ is high for Ic (54\%), Pd (50\%), C (45\%), Ig (44\%) and Sp (37\%). Furthermore the fraction of $\mathrm{L}$ is extremely high for Sk $(92 \%)$, and fairly high for Sp (60\%), C (48\%), Pd (38\%) and Pr (29\%), while it is low for Ic (13\%) and Ig (2\%).

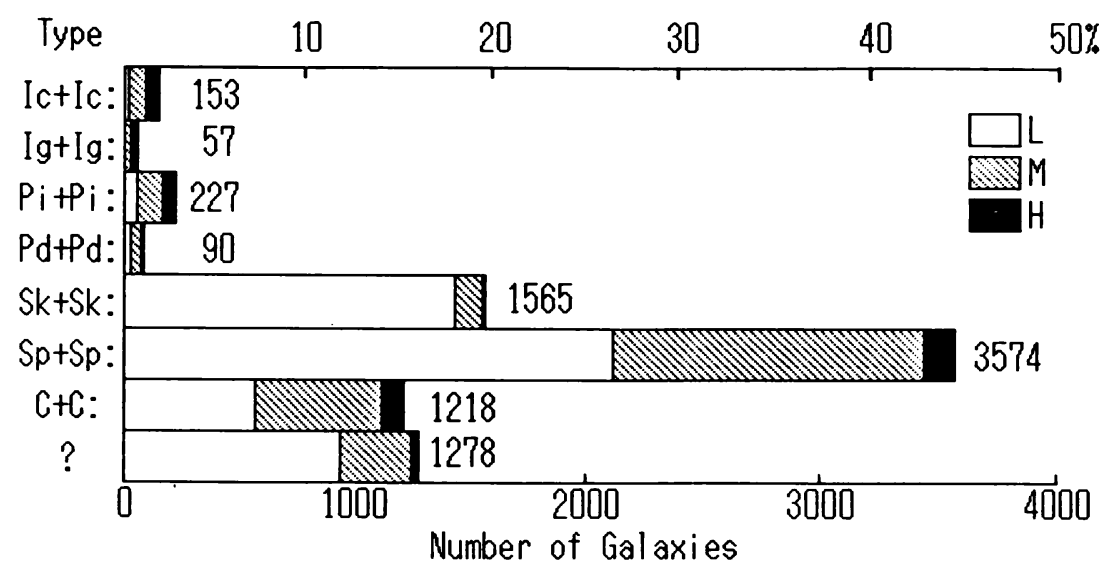

Figure 1. Frequency distribution of 8,162 KUGs in respect of morphological type and UV-excess degree. 


\section{References}

Noguchi, T., Maehara, H. and Kondo, M., 1980. Ann. Tokyo Astron. Obs., 2nd Ser., 18, 55.

Takase, B. and Miyauchi-Isobe, N., 1993a. Publ. Natl. Astron. Obs. Japan., (Catalogue 17) 3, 21.

Takase, B. and Miyauchi-Isobe, N., 1993b. Publ. Natl. Astron. Obs. Japan, (Catalogue 18) 3, 169.

Takase, B., Noguchi, T. and Maehara, H., 1983. Ann. Tokyo Astron. Obs., 2nd Ser., 19, 440. 